\title{
Use of anaerobic culture for the improved isolation of Streptococcus pneumoniae
}

\author{
R. HOWDEN
}

From the Bacteriology Department, Children's Hospital, Sheffield

SYNOPSIS The following report compares the results of aerobic and anaerobic cultivation for the primary isolation of Streptococcus pneumoniae (the pneumococcus) from the respiratory tract of children. Of 414 specimens of respiratory tract secretions cultured, $65(15.7 \%)$ yielded pneumococci; $31(47 \cdot 7 . \%)$ grew both aerobically and anaerobically, but $34(52 \cdot 3 \%)$ strains were isolated only from the anaerobic culture. Pneumococci cultured anaerobically with added carbon dioxide characteristically produce large mucoid colonies which are more easily distinguished than the 'normal' colonies commonly seen in the mixed flora isolated from respiratory sites. This advantage justifies the inclusion of anaerobic culture when attempting to isolate Str. pneumoniae from clinical material.

The routine procedure for the bacterial examination of respiratory tract swabs and secretions in this and many other clinical service laboratories rarely includes anaerobic culture. Recently, anaerobic cultivation has been improved by the use of a gas mixture of hydrogen and carbon dioxide instead of hydrogen alone, and by frequent control of the anaerobic jar's function (Collee et al, 1971; 1972). Following the introduction of these improvements, we noticed that pneumococci were more commonly isolated than formerly from respiratory specimens on primary anaerobic culture. The isolation of anaerobic pneumococci has previously been noted (Bolognesi, 1907; Smith, 1936), and, similarly, carbon dioxide has long been recognized as essential for the growth of a number of strains (Valley and Rettger, 1927; Auger, 1939; Fleming, 1941; Kempner and Schlayer, 1942), but the routine use of anaerobiosis with increased carbon dioxide has not previously been investigated. In this laboratory anaerobic strains of pneumococci appeared to be more common than earlier papers suggested, and consequently a comparison of the relative merits of aerobic and anaerobic culture was made.

\section{Material and Methods}

SPECIMENS

Throat swabs, nasal swabs, and occasionally sputa from children admitted with suspected respiratory

Received for publication 17 July 1975. infections were examined along with nasal swabs from an unselected group of children who were sampled for a variety of reasons not associated with respiratory tract infections. During normal laboratory hours, swabs were cultured as received, at varying time intervals after sampling. Outside these hours, swabs were immediately placed into Amies transport medium (Difco) and stored at room temperature overnight before being sent to the department. In all, 414 specimens received in a six-week period (October to December 1973) were examined.

\section{PATIENTS}

These were not selected by age or sex and showed a random age distribution, ranging from a few days to 12 years.

\section{CULTURE PROCEDURE}

All swabs were inoculated onto two Columbia base $N$ blood agar plates and a heated blood agar plate (Lab M Ltd, Salford, Lancs). All plates were less than 2 days old but may have been stored at $4^{\circ} \mathrm{C}$ overnight before use. The plates were incubated overnight at $37^{\circ} \mathrm{C}$. One of the blood plates and the heated blood plate were incubated aerobically $\Phi$ without $\mathrm{CO}_{2}$, and the other blood plate was incu-? bated anaerobically with $\mathrm{CO}_{2}$. Baird and Tatlock anaerobic jars were used. The detailed procedure for $\frac{}{\mathbb{D}}$ the setting up of the jars (hydrogen $90 \%$, carbon $\stackrel{?}{\stackrel{P}{P}}$ dioxide $10 \%$ was essentially that described by $\stackrel{\mathbb{Q}}{2}$ Collee et al (1972). As recommended by Collee? (personal communication) a culture of Pseudo- 8 
monas aeruginosa on Columbia nutrient agar, without blood, was included in each jar as a negative control of jar function.

Next morning all plates were removed, the control plate was checked, the tests were examined, and the results recorded independently by two experienced technicians, the aerobic plates being read by one and the anaerobic plate by the other.

The identity of suspected pneumococcal colonies was confirmed by microscopy, Gram's stain, and their sensitivity to ethyl hydrocuprein hydrochloride (Optochin) (Scientific Hospitals Supplies) at $37^{\circ} \mathrm{C}$ overnight. Initially, 'anaerobic' strains were tested against Optochin anaerobically but as minimal zones of lysis were produced, the presumptive identity of these strains was confirmed by their bile solubility (Lund, 1959). Soon it was realized that the anaerobic strains grew well aerobically on first subculture and that zones around the Optochin discs on aerobic culture were the same as those of the 'aerobic' strains. Bile solubility for identification was therefore replaced by Optochin sensitivity aerobically; the earlier 'anaerobic' cultures were retested aerobically.

In a number of strains the effect of various conditions for growth was examined.Thus cultures were compared on Columbia blood agar plates, heated Columbia blood agar plates, and Columbia agar without blood. Plates were incubated at $37^{\circ} \mathrm{C}$ overnight as follows: aerobically, aerobically with added $\mathrm{CO}_{2}$, anaerobically in hydrogen, and anaerobically in hydrogen with added $\mathrm{CO}_{2}$. Before being inoculated, two drops of purified catalase (Sigma) ( $1 \mathrm{mg} / \mathrm{ml}$ sterile solution) was spread over half of each plate to ascertain its effect on the production of hydrogen peroxide by the pneumococcus.

\section{Results}

Of the 414 specimens examined, $65(15.7 \%)$ yielded pneumococci; only $31(47.7 \%)$ of these grew aerobically on primary culture. That is to say, $34(52.3 \%)$ strains were obtained only by anaerobiosis with added $\mathrm{CO}_{2}$.

All those strains which initially had grown only anaerobically grew aerobically on subculture despite having failed to do so when first isolated.

It was not possible to quantitate the pneumococci

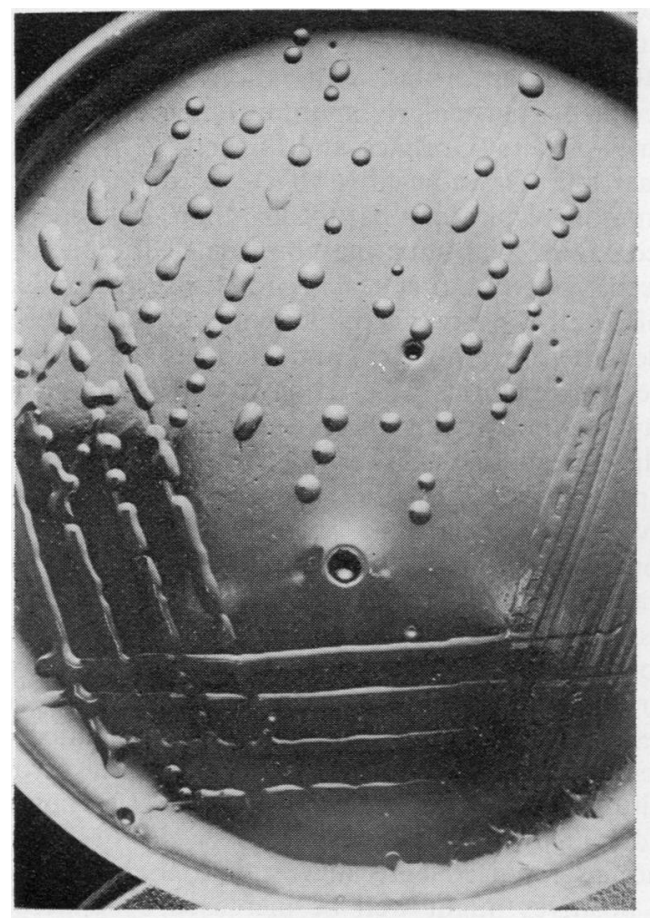

a

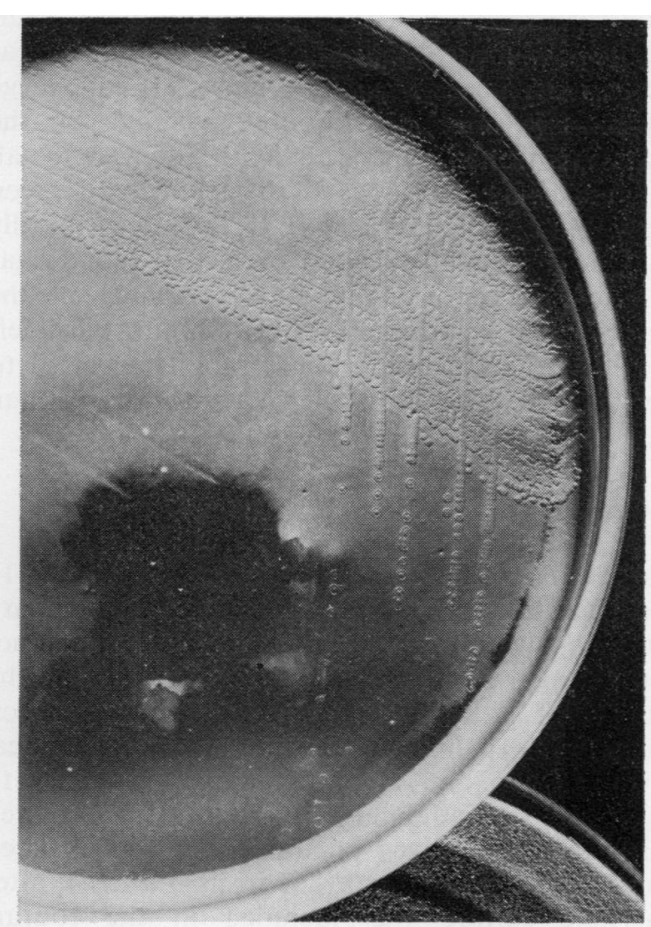

b

Figure A comparison of the colonial appearance of Streptococcus pneumoniae on Columbia blood agar plates after 18 hours' incubation at $37^{\circ} \mathrm{C}(\times 2):($ a) anaerobic culture with added carbon dioxide; (b) aerobic culture (without added carbon dioxide). 
isolated from each specimen as, generally, only swabs were received. Although it appeared that the number of colonies isolated from a specimen was greater on anaerobic than aerobic culture, this may simply have been because the marked difference in colonies made recognition on anaerobic plates so much easier. Strains cultured anaerobically, whether of the aerotolerant or initially non-aerotolerant type, almost always had a characteristic grey, watery or mucoid colony ( 2 to $3 \mathrm{~mm}$ diameter) but on aerobic culture colonies were of the plateau, socalled 'draughtsman' type (1 $\mathrm{mm}$ diameter) (see figure).

The growth comparison plates gave a series showing that anaerobic culture with $10 \% \mathrm{CO}_{2}$ produced the highest yield and the largest colonies, anaerobic cultures without $\mathrm{CO}_{2}$, aerobic culture with $\mathrm{CO}_{2}$, and aerobic culture without $\mathrm{CO}_{2}$ giving progressively less colonies and of the smaller type. Aerobically the yield on Columbia blood agar was greater than that on heated blood agar, which in turn was better than on Columbia agar without blood.

Catalase had little effect on the growth on blood agar or heated blood agar incubated aerobically, it was necessary for growth on Columbia agar without blood aerobically, but was not required when incubated anaerobically. Colonies on heated blood agar plates incubated anaerobically plus $\mathrm{CO}_{2}$ gave large grey moist colonies without 'greening' of the medium, contrasting sharply with the very small colonies with large areas of bleaching and green haloes produced aerobically. If the anaerobically incubated cultures on blood or heated blood agar were left at room temperature, greening of the medium occurred within one to two hours; when left overnight, the mucoid colonies rapidly reverted to large-scale versions of the classic draughtsman colonies.

\section{Discussion}

It is clear from the results of this small series of 414 specimens that the omission of anaerobic primary cultivation would have resulted in many pneumococci being missed. A retrospective study of the isolation of Str. pneumonia from respiratory specimens examined in the laboratory during identical periods before and after this survey (January to August 1973 and January to August 1974) showed that before the survey pneumococci were isolated from $5 \%$ of the specimens ( 267 strains) whereas after anaerobic culture was included in the routine procedure $8.2 \%$ gave positive results (435 strains). Whereas these two groups include all respiratory specimens received, the survey was limited to those with a high expectation of pneumococcal isolation; as such they illustrate the advantage of anaerobic culture with $\mathrm{CO}_{2}$ as part of the routine examination of respiratory specimens.

The mechanism of the production of large mucoid pneumococcal colonies anaerobically is not clear but is probably a product of a number of events. These include the stimulation of DNA synthesis, the shortening of the lag phase of growth (Walker, 1932; Repaske et al, 1974), and the inactivation of some of the toxic products either on the swab or present in the medium, but perhaps the most important is a reduction of the potency of the pneumococcal autolysin. Holt (1962) showed that autolysis of the pneumococcus resulted from the formation of hydrogen peroxide as a consequence of the organism's lack of a catalase system.

A reduction of hydrogen peroxide production on anaerobic growth with therefore little need for catalase only partly explains the results of this study. Catalase when added to aerobic cultures did not increase the size or alter the type of colony produced on blood agar plates but did act as a growth stimulator in the absence of blood. In one strain, $\mathrm{CO}$ accentuated the effect of anaerobiosis in that incubation in hydrogen alone gave small colonies whereas, when $\mathrm{CO}_{2}$ was included, the colonies produced were of the large mucoid type.

Routine anaerobic cultivation of respiratory specimens, particularly sputum, has been urged by some workers (Gorbach and Bartlett, 1974) because anaerobic Gram-negative bacteria may be more common respiratory pathogens than is generally recognized. The same may be true of the pneumococcus, but our first task is to devise methods to isolate all the organisms present and then to consider their significance and, if necessary, devise selective methods. 'Selection by default'-which may be what we have been doing up to now-is no help to anyone. Since the introduction of the improved culture method 'anaerobic' pneumococci have also been isolated on a number of occasions from cerebrospinal fluids, blood cultures, and eye swabs. The method has been especially useful in ensuring the growth of the small numbers of pneumococci which may survive in specimens from partially treated cases. The method may overcome the problem that only about half of the pneumococci seen in stained films of sputa grow on aerobic culture (Lepow et al, 1968; Fiala, 1969; Tugwell and Greenwood, 1975; Nicholls et al, 1975). The findings reported here suggest that the proportion of positives might well be increased by anaerobic culture of such specimens. The importance of such an increased isolation would be mainly in the diagnosis of meningitis and septicaemia, where the pneumococcus is clearly pathogenic. 
I wish to acknowledge the invaluable advice given by Professor McEntegart, Department of Medical Micribiology, University of Sheffield, and Professor J. A. Collee, Bacteriology Department, University of Edinburgh Medical School, in preparing this manuscript. I would also like to thank Miss Susan Horsey who typed it.

\section{References}

Auger, W. J. (1939). A new method of culturing sputum on solid media using carbon dioxide for the isolation of pneumococci. Brit. J. exp. Path., 20, 439-442.

Bolognesi, G. (1907). Die Anärobiose des Fränkelschen Diplococcus in Beziehung zu einer seiner pathogenen Eigenschaften. Zbl. Bakt. I. Abt. Orig., 43, 113-118.

Collee, J. G., Rutter, J. M., and Watt, B. (1971). The significant viable particle: a study of the subculture of an exacting sporing anaerobic. J. med. Microbiol., 4, 271-288.

Collee, J. G., Watt, B., Fowler, E. B., and Brown, R. (1972). An evaluation of the Gaspak system in the culture of anaerobic bacteria. J. appl. Bact., 35, 71-82.

Fiala, M. (1969). A study of the combined role of viruses, mycoplasmas and bacteria in adult pneumonia. Amer. $J$. med. Sci., 257, 44-51.

Fleming, A. (1941). A pneumococcus which required $\mathrm{CO}_{2}$ for its growth. Lancet, 1, 110.
Gorbach, S. L. and Bartlett, J. G. (1974). Anaerobic infections. Pt. II (Chest and miscellaneous infections). New Engl. J. Med., 290, 1237-1245.

Holt, L. B. (1962). The culture of Streptococcus pneumoniae. J. gen. Micribiol., 27, 327-330.

Kempner, W. and Schlayer, C. (1942). Effect of $\mathrm{CO}_{2}$ on the growth rate of the pneumococcus. J. Bact., 43, 387-396.

Lepow, M. L., Balassanian, N., Emmerich, J., Roberts, R. B., Rosenthal, M. S., and Wolinsky, E. (1968). Interrelationships of viral, mycoplasmal, and bacterial agents in uncomplicated pneumonia. Amer. Rev. resp. Dis., 97, 533-545.

Lund, E. (1959). Diagnosis of pneumococci by the optochin and bile tests. Acta path. microbiol. Scand., 47, 308-315.

Nicholls, A. C., Pease, P. E., and Green, I. D. (1975). Agglutinin response to bacterial infection in acute exacerbations of chronic bronchitis. J. clin. Path., 28, 279-283.

Repaske, R., Repaske, A. C., and Mayer, R. D. (1974). Carbon dioxide control of lag period and growth of Streptococcus sanguis. J. Bact., 117, 652-659.

Smith, F. (1936). Anaerobic pneumococcus. Brit. J. exp. Path., 17, 329-334.

Tugwell, P. and Greenwood, B. M. (1975). Pneumococcal antigen in lobar pneumonia. J. clin. Path., 28, 118-123.

Valley, G. and Rettger, L. F. (1927). The influence of carbon dioxide on bacteria. J. Bact., 14, 101-137.

Walker, H. H. (1932). Carbon dioxide as a factor affecting lag in bacterial growth. Science, 76, 602-604. 